\title{
Role of activation of the coagulation system in the pathogenesis of urticaria
}

\author{
Taro Yasuma ${ }^{1}$, Corina D’Alessandro-Gabazza ${ }^{1}$, Tetsu Kobayashi ${ }^{1}$, John Morser $^{2}$, and \\ Esteban Gabazza ${ }^{1}$ \\ ${ }^{1}$ Mie Daigaku Daigakuin Igakukei Kenkyuka Igakubu \\ ${ }^{2}$ Stanford University
}

July 6, 2021

\section{Role of activation of the coagulation system in the pathogenesis of urticaria}

Taro Yasuma, ${ }^{1}$ Corina N. D’Alessandro-Gabazza, ${ }^{1}$ Tetsu Kobayashi, ${ }^{2}$ John Morser, ${ }^{3}$ Esteban C Gabazza. ${ }^{1 *}$

${ }^{1}$ Department of Immunology, ${ }^{2}$ Department of Pulmonary and Critical Care Medicine, Mie University Faculty and Graduate School of Medicine, and Mie University Hospital, Edobashi 2-174, Tsu, Mie 514-8507, Japan. ${ }^{3}$ Division of Hematology, Stanford University School of Medicine, 291 Campus Drive, Stanford, CA 94305, United States

*Correspondence: Esteban C Gabazza, MD, PhD, Department of Immunology, Mie University Graduate School of Medicine, Edobashi 2-174, Postal Code 514-8507, Tsu-city, Mie, Japan. Tel.: +81 59231 5017; fax: +8159231 5225.

E-mail:gabazza@doc.medic.mie-u.ac.jp

Word count: 590

To the Editor,

We read with much interest the review article recently published by Mostmans et al. (1). The work is an excellent compilation of studies on alterations of vascular endothelial cell-associated molecules and coagulation factors in urticaria. We want to congratulate the authors for the completion of this comprehensive review. However, despite mounting evidence, we were surprised that the authors commented in the discussion section that the "implication of a prothrombotic state in chronic urticaria patients is still under debate since both depressed coagulation activity and normal plasma coagulation factors have also been reported." A comparative look at the coagulation profile used in all studies described in Table 5 of the article shows that researchers describing upregulation of coagulation parameters used well-recognized markers of coagulation activation, whereas researchers reporting "normal plasma coagulation factors" used less sensitive assay or non-canonical markers of clotting activation (1). In addition, the fact that one or two markers among a set of coagulation parameters remain normal or low in urticaria patients compared to a control group does not necessarily mean there is no activation of coagulation. For example, there was no significant change in the plasma levels of activated factor VII but there were increased plasma levels of D-dimer or thrombinantithrombin complex in the same urticaria patients compared to controls $(2,3)$. Another important issue that needs to be considered is the study design. For example, the study reported by Takahagi et al. had no control group (4). They considered the plasma levels of D-dimer and fibrin-degradation products as normal or high merely based on a cut-off line. Although not all patients showed levels of D-dimer or fibrin-degradation products higher than the cut-off value, the authors found significantly increased plasma concentrations of D-dimer and fibrin degradation products in patients with severe urticaria compared to patients with 
moderate/slight disease and, importantly, that the levels of those markers increased during exacerbations compared with before or after (4). The material or sample used to measure hemostatic parameters is also an important source of bias. For example, Zhu et al. reported lower plasma levels of soluble tissue factor in urticaria patients than in controls (3). However, most studies have described significantly increased tissue factor expression on the cell surface of leukocytes in skin lesions from urticaria patients compared to controls $(5,6)$. This is understandable because tissue factor is generally expressed as a cell membranebound protein, at particular sites of tissue injury, while just a small fraction of it is released as a soluble factor. Other issues that were not considered that are important for conclusions about previous studies are the study population size, the inclusion of historic or concurrent controls, the use of validated assays and whether the results were confounded by treatment regimens. In addition to lessons learned from this review by Montmans et al., other proofs of the involvement of the coagulation cascade in urticaria include reports showing the beneficial effect of anticoagulants (e.g., heparin, warfarin, nadroparin) in patients with refractory urticaria or unresponsive to antihistamines, and reports showing that the plasma levels of Ddimer can predict clinical response to therapy with antihistamines or anti-IgE monoclonal antibody $(1,5)$. What remains debatable is whether IgE antibodies against tissue factor with concomitant dysfunction of the endothelial cell-mediated thrombomodulin/protein C anticoagulant pathway plays a major role in the mechanism of coagulation activation in urticaria patients (5). Taken together, we believe that there is robust evidence in the literature supporting the role of the coagulation cascade activation and consequent deposition of fibrin in the affected areas in the pathogenesis of urticaria.

\section{Author Contributions}

T.Y., C.N.D-G, T.K., J.M, and E.C.G. wrote, edited and approved the draft of the letter:

\section{Conflict of interest}

All authors declared no conflict of interest regarding this letter. T.K. reports grants and personal fees from Chugai, grants and personal fees from Pfizer, grants and personal fees from ONO, grants from TAIHO, grants and personal fees from Boehringer Ingelheim, grants and personal fees from Eli Lilly, outside the submitted work. E.C.G. reports grants from Shionogi Pharmaceutical Inc., grants from Astellas Pharmaceuticals Inc., grants from Aqua Therapeutics, outside the submitted work.

\section{References}

1. Mostmans Y, De Smedt K, Richert B, Elieh Ali Komi D, Maurer M, Michel O. Markers for the involvement of endothelial cells and the coagulation system in chronic urticaria: A systematic review. Allergy 2021.

2. Takeda T, Sakurai Y, Takahagi S, Kato J, Yoshida K, Yoshioka A, et al. Increase of coagulation potential in chronic spontaneous urticaria.Allergy 2011;66 :428-433.

3. Zhu H, Liang B, Li R, Li J, Lin L, Ma S, et al. Activation of coagulation, anti-coagulation, fibrinolysis and the complement system in patients with urticaria. Asian Pac J Allergy Immunol2013;31 :43-50.

4. Takahagi S, Mihara S, Iwamoto K, Morioke S, Okabe T, Kameyoshi Y, et al. Coagulation/fibrinolysis and inflammation markers are associated with disease activity in patients with chronic urticaria. Allergy2010;65 :649-656.

5. Asero R, Marzano AV, Ferrucci S, Cugno M. Elevated baseline D-dimer plasma levels are associated with a prompt response to omalizumab in patients with severe CSU. J Allergy Clin Immunol Pract2017;5 :1740-1742.

6. Saito R, Yanase Y, Kamegashira A, Takahagi S, Tanaka A, Uchida K, et al. Increase of tissue factor expression on the surface of peripheral monocytes of patients with chronic spontaneous urticaria. Allergy2020;75 :971-974.

\section{Legend for Figure 1}


Figure 1. Activation of the coagulation system in chronic urticaria. Mast cells release inflammatory mediators including histamine after degranulation leading to activation of endothelial cells and eosinophils. Activated endothelial cells and eosinophils express tissue factor initiating coagulation activation after binding to activated factor VII (FVIIa) generating thrombin. Thrombin induces activation of platelets, cleaves fibrinogen, recruits inflammatory cells, activates mast cells and endothelial cells by binding to and cleaving protease-activated receptor (PAR-1) leading to increased vascular permeability with extravascular spillover of inflammatory mediators. EOS, eosinophils; MC, mast cells; NEU, neutrophils; F1+2, prothrombin fragment $1+2$; FDP, fibrin degradation products; PL, phospholipids.
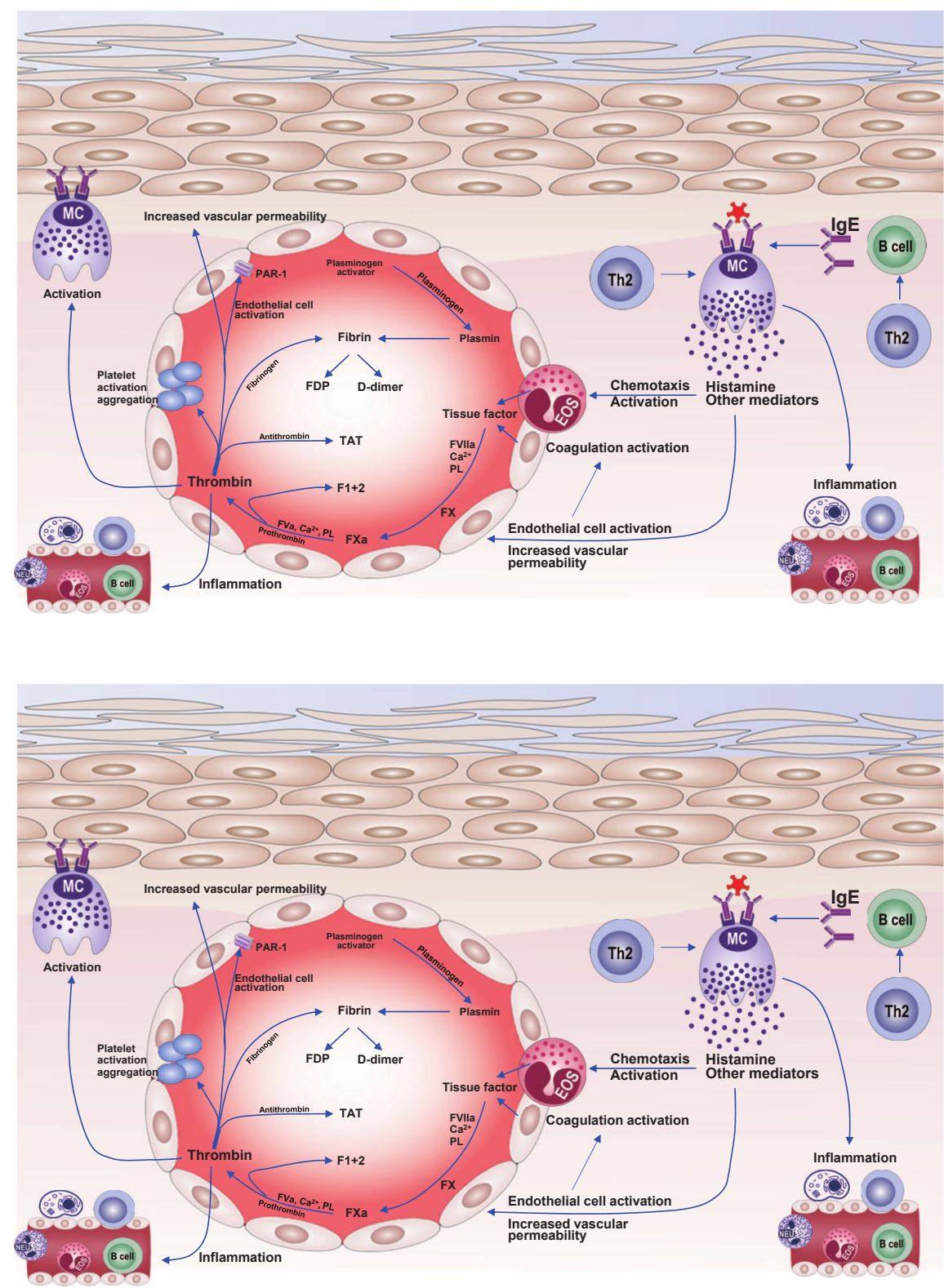